\title{
Populations of WC and WN stars in Wolf-Rayet galaxies: new constraints on starburst parameters
}

\author{
Thierry Contini ${ }^{1}$, Daniel Schaerer ${ }^{2}$, and Daniel Kunth ${ }^{3}$ \\ ${ }^{1}$ European Southern Observatory, Garching, Germany \\ ${ }^{2}$ Observatoire Midi-Pyrénées, Toulouse, France \\ ${ }^{3}$ Institut d'Astrophysique de Paris, France
}

\begin{abstract}
Detection and analysis of Wolf-Rayet star populations in young starbursts is a powerful tool to reveal the properties of the star-forming events. We present new high $\mathrm{S} / \mathrm{N}$ optical spectra of a sample of well-known WR galaxies, in which we detected for the first time the spectral signature of WC stars. The detailed comparison of WN and WC star populations derived in these galaxies, with the most recent model predictions, allows us to put tight constraints on starburst parameters, such as the age and duration of the burts, and the IMF.
\end{abstract}

\section{Introduction}

Starbursts play a major role in the global process of galaxy formation and evolution. A better knowledge of their intrinsic properties (duration, IMF, etc) in nearby starburst galaxies is a key issue to follow the evolution of the large population of star-forming galaxies observed up to redshift $z \simeq 4$ (e.g., Steidel et al. 1999). The so-called 'Wolf-Rayet galaxies' may be the ideal laboratories to derive starburst properties since these objects harbour the most massive stars known, O stars and their descendent WR stars, which allow us to probe the upper part of the IMF and the youngest stellar populations.

Since the initial compilation of Conti (1991) listing 37 galaxies, the number of known WR galaxies has grown rapidly to about 130 in the last compilation of Schaerer, Contini \& Pindao (1999, hereafter SCP99). While most of them fall in the category of 'classical' star-forming galaxies (HII galaxies or Starburst Nucleus Galaxies), broad WR emission lines have also been detected in more 'exotic' objects like Seyfert 2 galaxies (e.g., Heckman et al. 1997; Kunth \& Contini, these Proceedings) or giant $\mathrm{cD}$ galaxies, located in the centre of galaxy clusters with strong cooling flows (Allen 1995).

The presence of a large number of WR stars has already provided interesting informations about star formation in WR galaxies, indicating recent $(\$ 10 \mathrm{Myr})$ star-forming events and the existence of massive stars (e.g., Vacca \& Conti 1992, hereafter VC92). But in practice, the detection of the broad emission line He II $\lambda 4686$, attributed mainly to WR stars of WN subtype, was often simply used. The strongest emission line of WC stars, $\mathrm{C}$ IV $\lambda 5808$, has quite rarely been observed so far in WR galaxies. Although Conti (1991) claimed the absence of convincing evidence for carbon features in WR galaxies, there is no doubt about their existence anymore since about 56 objects showing both broad He II $\lambda 4686$ and C IV $\lambda 5808$ features are listed in SCP99. 
The existence of WC stars in WR galaxies, although possibly less numerous than WN stars, is indeed expected both from observations of WR populations in the Local Group (e.g., Massey \& Johnson 1998, hereafter MJ98), and from stellar evolution models (Maeder \& Meynet 1994). Surprisingly, the homogeneous, fairly high $\mathrm{S} / \mathrm{N}$ spectra from the sample of VC92 containing 12 regions with He II $\lambda 4686$ detections revealed only one C IV $\lambda 5808$ detection (in He2-10 A). If true, this would certainly contradict the predictions from synthesis models for young starbursts (Meynet 1995; Schaerer \& Vacca 1998, hereafter SV98), which otherwise compare well to observations in the Local Group (Maeder \& Meynet 1994). To verify if this apparent discrepancy really holds we have searched for WC stars in well-known WR galaxies (NGC 3049, He2-10, NGC 3125, NGC 5253, Tol 89). Previous accounts of our results are given in Schaerer et al. (1997) and Schaerer, Contini \& Kunth (1999, hereafter SCK99).

\section{Observations and spatial analysis}

Long-slit spectra of galaxies were obtained on the nights of 1995 April 24-26 at the ESO $2.2 \mathrm{~m}$ telescope. The data were acquired with the EFOSC2 spectrograph which gives a spectral coverage of $4400-6500 \AA$ with a resolution of $\sim 5 \AA$. The slit was oriented in order to cover regions with previous detections of WR stars. The total integration time for each galaxy ranges from 80 to $120 \mathrm{~min}$. The seeing was relatively stable during the observations with a mean spatial resolution of about $1^{\prime \prime}$. The procedure for data reduction is fully described in SCK99.

We used our long-slit spectroscopic observations to compare, for each galaxy, the spatial distributions of nebular and WR emission-line intensities and the stellar continuum emission. The results for each galaxy are given in SCK99; they can be summarized as follows. There is an offset between the distribution of nebular lines and stellar continuum in He2-10 and Tol 89. The WR emission features follow the stellar continuum with no significant shift between He II $\lambda 4686$ and C IV $\lambda 5808$. The spatial extension of WR features ranges from $\sim 60 \mathrm{pc}(\mathrm{NGC} 5253)$ to $\sim 270 \mathrm{pc}$ (He2-10 and NGC 5253). Two distinct WR regions are identified in NGC 3125 and NGC 5253.

\section{The Wolf-Rayet regions}

One-dimensional spectra were extracted corresponding to the different regions where spectral signatures from WR stars have been detected. Normalized spectra showing the region around the 'blue' and 'red' WR bumps are given in Fig. 1. Several broad emission lines due to WR stars have been detected over the wavelength range covered by our spectra: the blend of $\mathrm{N}$ III $\lambda 4640$ and $\mathrm{C}$ III $\lambda 4650$, He II $\lambda 4686$, and $\mathrm{C}$ IV $\lambda 5808$. Equivalent widths $(E W s)$ of these emission lines are listed in Table 1, together with the basic nebular parameters (metallicity, extinction, etc).

One of the main results of these observations is the unambiguous detection of broad (FWHM $\simeq 50-90 \AA$ ) C IV $\lambda 5808$ emission in all WR regions of the observed galaxies (see right panel of Fig. 1), which clearly indicates the presence of $W C$ stars in these regions. Whereas all the objects were previously known to show the broad WR bump around $4700 \AA$, our spectra are the first to show the red 

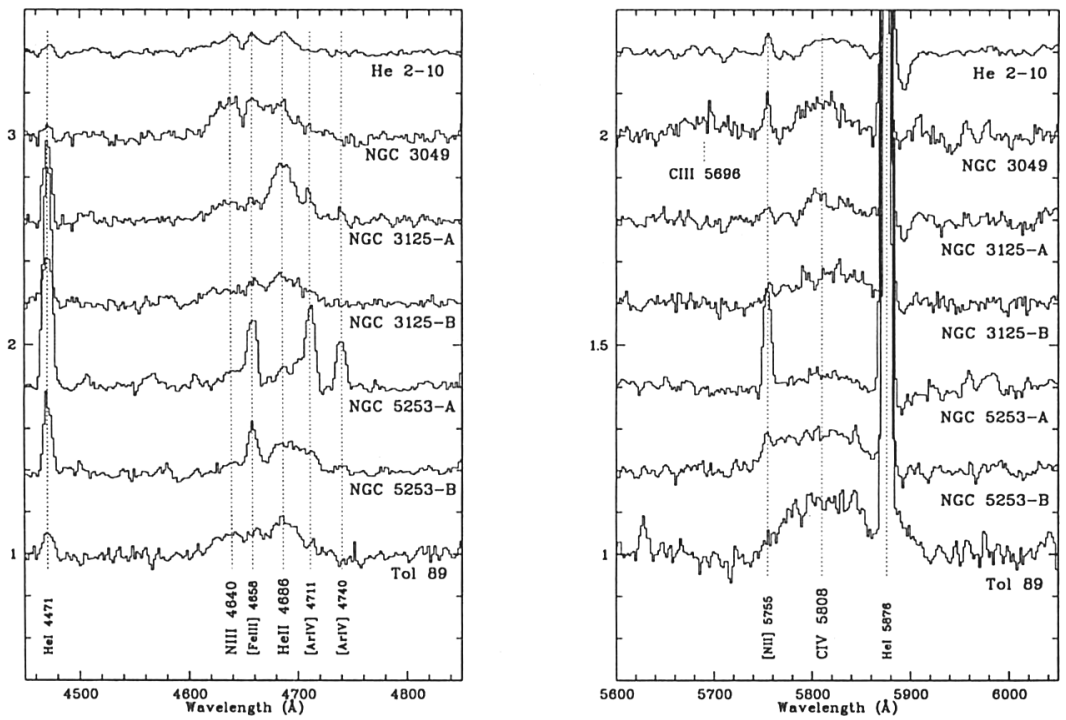

Figure 1. Spectra of galaxies showing the 'blue' (left) and 'red' (right) WR bumps.

WR bump around $5800 \AA$. As already noted (Schaerer et al. 1997, SCK99), a sufficiently high $\mathrm{S} / \mathrm{N}(\gtrsim 40)$ is required for the detection of such a $\mathrm{C}$ IV $\lambda 5808$ line in integrated spectra of WR galaxies. This explains why this line has remained undetected in previous ground-based spectroscopical studies, except for He2-10 (VC92).

In all our seven emission-line regions we thus find signatures of both WN and WC stars. The dominant WR subtypes in each region can be constrained using measured line widths and intensity ratios between WR emission lines (see SCK99 for a full description). All regions (except the high-metallicity galaxy NGC 3049) contain a mixed population of WNL, and early WC and/or WO34 stars. In NGC 3049 , the detection of $\mathrm{C}$ III $\lambda 5696$ indicates the predominance

Table 1. The broad Wolf-Rayet emission lines

\begin{tabular}{lrrrrrrr}
\hline line/galaxy & He2-10 & NGC 3049 & \multicolumn{2}{c}{ NGC 3125 } & \multicolumn{2}{c}{ NGC 5253 } & Tol 89 \\
\cline { 3 - 6 } & & \multicolumn{7}{c}{ A } & B & A & B & \\
\hline$E W(\mathrm{H} \beta)[\AA]$ & $23 \pm 3$ & $44 \pm 3$ & $93 \pm 9$ & $70 \pm 7$ & $269 \pm 23$ & $112 \pm 10$ & $68 \pm 17$ \\
$(\mathrm{O} / \mathrm{H})\left[\mathrm{Z}_{\odot}\right]$ & 0.42 & 1.20 & 0.17 & 0.20 & 0.20 & 0.20 & 0.25 \\
$C_{\mathrm{H} \beta}$ & 0.56 & 0.23 & 0.40 & 0.64 & 0.44 & 0.20 & 0.18 \\
\hline \multicolumn{7}{c}{$E W[\AA]$ of broad emission lines from Wolf-Rayet stars } \\
\hline N III $\lambda 4640$ & $2.7 \pm 0.5$ & $5.2 \pm 0.5$ & $3.2 \pm 0.5$ & $2.8 \pm 1.0$ & $2.1 \pm 1.3$ & $1.0 \pm 0.5$ & $3.4 \pm 0.5$ \\
+ C III $\lambda 4650$ & & & & & & \\
He II $\lambda 4686$ & $2.3 \pm 0.5$ & $5.6 \pm 0.5$ & $7.3 \pm 1.0$ & $2.8 \pm 0.5$ & $3.0 \pm 0.7$ & $3.3 \pm 1.1$ & $6.1 \pm 0.5$ \\
C III $\lambda 5696$ & $\ldots$ & $2.0 \pm 0.5$ & $\ldots$ & $\ldots$ & $\ldots$ & $\ldots$ & $\ldots$ \\
C IV $\lambda 5808$ & $1.5 \pm 0.5$ & $4.7 \pm 0.6$ & $3.1 \pm 0.4$ & $6.2 \pm 1.0$ & $2.5 \pm 0.6$ & $6.0 \pm 1.1$ & $11.9 \pm 1.5$ \\
\hline
\end{tabular}


of WCL stars. The presence of intermediate-type WN/WC stars cannot be excluded.

\section{Populations of Wolf-Rayet and O-type stars}

The approximate number of WN, WC, and O-type stars derived from the observed emission lines are listed in Table 2. The number of WN and WC stars is calculated from the luminosity of the WR emission lines, assuming that the dominant contributors to the broad He II $\lambda 4686$ and C IV $\lambda 5808$ lines are WNL and WC4 stars, respectively (see previous section). Under the condition of case $B$ recombination and assuming that all the ionizing photons emitted by the stars are absorbed by the gas, the total number of Lyman photons can be derived from the observed luminosity of the $\mathrm{H} \beta$ emission line. The number of $\mathrm{O}$ stars is thus estimated, taking into account the ionizing photon contribution from WR stars, the age of the stellar population and the IMF (see SCK99). The number of $\mathrm{O}$ stars found for the WR regions are between $\sim 500$ and 7000. The case of NGC 3049 is somewhat exceptional (see SCK99 for a discussion). According to the models, the bulk of the ionisation in this galaxy is provided by late $\mathrm{O}$ and/or $\mathrm{B}$ type stars and WR stars. Uncertainties on absolute number of WR and O stars reported in Table 2 are quite large. The main sources are the following (see SCK99 for details). There is a large uncertainty on the average luminosity of WNL stars in the He II $\lambda 4686$ line, and to a lesser extent of WCE stars in the CIV $\lambda 5808$ line. The dominant WC subtype is not well constrained. There may be a nebular contribution to the He II $\lambda 4686$ emission in NGC 3125-A and NGC 5253-A (see Fig 1). A significant fraction of ionizing photons may escape from Hil regions.

The number ratios $\mathrm{WR} / \mathrm{O}$ and $\mathrm{WC} / \mathrm{WN}$ are reported in Table 2 . We obtain WR/O number ratios of $\sim 0.03-0.4$ (and even larger for NGC 3049), systematically higher than the predictions for constant star formation at the appropriate metallicity (Maeder \& Meynet 1994), but within the range of instantaneous burst models with different IMF slopes (SV98). A trend of increasing WR/O ratios towards higher metallicity is found as expected (e.g., Meynet 1995). The number ratio of WC/WN stars is typically $0.2-0.4$, with no systematic variation with metallicity. For the low-metallicity objects $\left(Z \simeq 0.2 \mathrm{Z}_{\odot} ; \mathrm{NGC} 3125\right.$, NGC 5253, and Tol 89) the derived WC/WN ratio is larger than what is found in the Local Group (except IC 10) at similar metallicity (MJ98). At higher metal-

\begin{tabular}{lllll} 
Table 2. & Populations of Wolf-Rayet and O-type stars in WR regions \\
\hline axy burst & WN WC & O & WR/O WC/WN
\end{tabular}

\begin{tabular}{|c|c|c|c|c|c|c|c|}
\hline galaxy & & $\begin{array}{c}\text { age burst } \\
{[\mathrm{Myr}]}\end{array}$ & WN & $\overline{\mathrm{WC}}$ & O & $\overline{\mathrm{WR} / \mathrm{O}}$ & WC/WN \\
\hline $\mathrm{He} 2-10$ & & $\frac{1.5-6.0}{5.5-6}$ & $1100 \pm 520$ & $>250$ & $2450-4900$ & 0.37 & 0.23 \\
\hline NGC 3049 & & 5.5 & $510 \pm 240$ & $>170$ & & & 0.33 \\
\hline NGC 3125 & A & $4.5-5.0$ & $500 \pm 230$ & $>70$ & $3240-6470$ & 0.12 & 0.14 \\
\hline & B & $4.5-5.0$ & $530 \pm 250$ & $>200$ & $3450-6900$ & 0.14 & 0.38 \\
\hline NGC 5253 & A & 3.0 & $26 \pm 13$ & $>9$ & $960-1080$ & 0.03 & 0.35 \\
\hline & $\mathrm{B}$ & 5.0 & $27 \pm 13$ & $>10$ & 680 & 0.05 & 0.37 \\
\hline Tol 89 & & $4.5-5.0$ & $240 \pm 110$ & $>150$ & $640-1270$ & 0.41 & 0.62 \\
\hline
\end{tabular}


licities, He2-10 and NGC 3049 show, however, WC/WN ratios below the trend observed by MJ98.

The finding of a fairly constant $\mathrm{WC} / \mathrm{WN}$ ratio may seem surprising at first sight. Contrary to regions of constant star formation, likely representative of the Local Group samples, regions of short star formation could a priori show quite a large range in $\mathrm{WC} / \mathrm{WN}$, depending on the age of the starburst. However, as explained in SCK99, burst durations of $\gtrsim 2$ Myr already suffice to smooth out the $\mathrm{WC} / \mathrm{WN}$ ratio. This may well explain the small range of $\mathrm{WC} / \mathrm{WN}$ found for the low-metallicity objects. More surprising is the low WC/WN ratio of the remaining higher metallicity objects (He2-10 and NGC 3049) compared to the WC/WN value of MJ98 at a similar metallicity. Indeed, the probability of finding WC/WN ratios below the equilibrium value attained in regions of constant star formation should be quite small. However, if the number of WC stars is systematically underestimated by a factor of 3 , our observations may well all be larger than the observed WC/WN trend with $Z$. We conclude that the WC/WN ratios of the low-metallicity galaxies can be understood quantitatively with burst models of reasonably short but non-zero duration. Additional observations of WC and WN populations, especially for regions of higher metallicities, would be very helpful.

\section{Age, duration and IMF of starbursts}

One can try to constrain the main burst parameters (age, IMF, star formation history) by comparing the observed WR signatures to the predictions from the latest population synthesis models for young starbursts (SV98). We consider two basic free model parameters: the IMF slope and the duration of the starforming event. The metallicity $Z$ and the starburst age (from $E W(\mathrm{H} \beta)$ ) are adopted from the observations. A power law with a slope $\alpha$ is adopted for the IMF (in our notation $\alpha=2.35$ for a Salpeter IMF). The upper mass cutoff is generally set to $M_{\text {up }}=120 \mathrm{M}_{\odot}$. We consider burst models with different durations $\Delta t$ starting at time $t=0$, with the limiting case of an instantaneous burst $(\Delta t=0)$. SV98 models are based on the latest Geneva stellar evolution tracks and only single-star models are considered. A detailed discussion on the assumptions or uncertainties of the models is given in SCK99.

The comparison with the observations is performed in two ways. We both compare relative line intensities of the WR features with respect to the nebular $\mathrm{H} \beta$ emission (in short $\mathrm{WR} / \mathrm{H} \beta$ ), and equivalent widths of the WR lines. Observationaly the determination of these quantities can mainly be 'perturbed' by three effects:

(i) We do not count all the ionizing photons produced by massive stars. This may happen if the nebular emission from the HII region of interest is not entirely included in the slit, and/or if ionizing photons can escape from the HII region.

(ii) Stars and gas suffer from a different extinction.

(iii) An underlying older population contributes additional continuum light. 

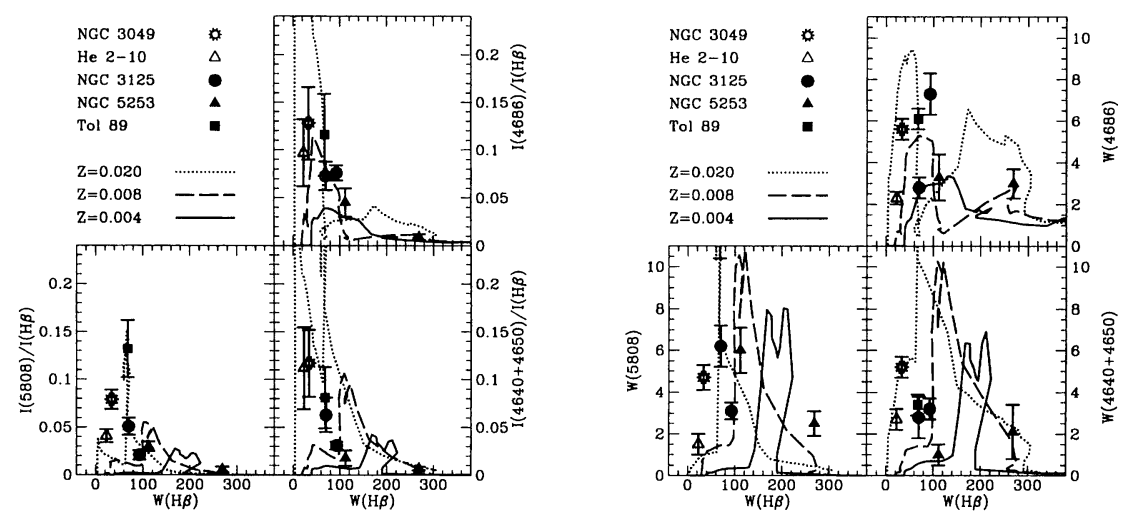

Figure 2. Observed vs. predicted WR line-intensities relative to $\mathrm{H} \beta$ (left) and WR equivalent widths (right).

The relative intensities $\mathrm{WR} / \mathrm{H} \beta$ are affected only by (i) and (ii). Effect (i) increases the relative $\mathrm{WR} / \mathrm{H} \beta$ intensities. The same holds for (ii) if the stellar light has less extinction than the gas ( $c f$. e.g., Calzetti 1997, Mas-Hesse \& Kunth 1999). The EWs are only affected by (iii), which decreases the observed value. We refer to SCK99 for a discussion on the quantitative importance of these effects. Given these potential difficulties both comparisons of relative line intensities $\mathrm{WR} / \mathrm{H} \beta$ and $\mathrm{WR}$ equivalent widths have been performed for all objects. Significant differences between the two methods likely indicate some difficulty with (i) and (iii). In this case the simplest meaningful comparison is between the observed WR equivalent widths and the maximum value predicted by the models (irrespectively of the age); it only depends on (iii).

In Fig. 2 we compare, for all the WR regions, the observed relative intensities and EWs of WR lines with the model predictions of SV98 at metallicities $Z=0.02$ (solar), 0.008, and 0.004 for an instantaneous burst with a Salpeter IMF. In both plots, $W(\mathrm{H} \beta)$ is used as a time indicator (decreasing $W$ with time) in the synthesis models. Comparison of the individual objects (classified by metallicity) with the model predictions can be summarized as follows.

\subsection{High-metallicity galaxies}

The predictions of the standard model (instantaneous burst, Salpeter IMF) are in agreement with the observed WR line strengths of the high-metallicity galaxies, particularly for NGC 3049. For He2-10, the observed EWs are considerably smaller than the maximum value predicted by the models, but the relative line intensities are close to the predicted maximum. This difference could be attributed to the shift between gaseous and stellar emission (see sect. 2.).

\subsection{Low-metallicity galaxies}

For the low-metallicity objects, the comparison between data and model predictions is much more complex. Only some of the observed WR line intensities and EWs lie well on the predicted model curves plotted in Fig. 2. For the con- 
sidered objects we have a total of 15 measurements of broad emission lines (all lines and all regions). The relative line intensity of $7 \mathrm{WR}$ regions is above the maximum value predicted by the standard model. On the other hand the majority (13 of 15) of the EWs are well in the range of the model predictions. The two remaining cases are $W(\mathrm{He}$ II $\lambda 4686)$ of NGC 3125-A and Tol 89, which could well be overestimated due to nebular contamination and contributions from other lines in the blended region, and an exceptionally large C IV $\lambda 5808$ in Tol 89. The uncertainties affecting the observed quantities are fully discussed in SCK99. Only for Tol 89 we have direct evidence that the displacement between gas and stars is likely of importance (see sect.2.). For this galaxy we measure an extraordinarily strong C IV $\lambda 5808$ emission (see Table 1) which exceeds the values shown in Fig. 2. We have no simple explanation for this strong emission. Other observations are required to confirm the measurements for Tol 89.

One possibility to explain the observations of some regions with large $\mathrm{WR} / \mathrm{H} \beta$ intensity ratios and large $W(\mathrm{WR})$ may e.g., be a flatter IMF. Models with different burst durations and IMF have been tested in SCK99 (their fig. 12). These show that, within reasonable assumptions for the IMF slope, the duration of the star formation episode is limited to $\Delta t \lesssim 2-4 \mathrm{Myr}$. What can be said about the IMF slope? As mentioned earlier the observations of the WR features in NGC 3049, He2-10, and NGC 5253 are quite compatible with a Salpeter IMF. However, the large observed values of $W(4686)$ in NGC 3125-A and Tol 89, and $W(5808)$ in Tol 89 may require more WR stars. This can be obtained by invoking a flatter IMF, which increases the WR equivalent widths. However, we note that intrinsically the model predictions for He II $\lambda 4686$ are not very sensitive to changes of the IMF slope. More IMF-sensitive are the features of WC stars, which descend on the average from more massive predecessors than WN stars and from a narrower range of initial masses. Although the standard models show some deficiencies, it is difficult to claim significant differences with respect to a Salpeter IMF (see SCK99 for details). We therefore conclude that within the uncertainties our observations are compatible with a Salpeter IMF. Although we cannot exclude this possibility, no clear case requiring a significantly flatter IMF is found. Much steeper IMF slopes may, however, not be compatible with our data.

\section{Conclusions}

The main result of this work is the unambiguous detection of WC stars in five previously known WR galaxies, which brings to $\sim 54$ the total number of extragalactic objects known to harbour both WN and WC stars (cf. SCP99). The relative weakness of $\mathrm{C}$ IV $\lambda 5808$ compared to He II $\lambda 4686$, and its larger width requires sufficiently high $\mathrm{S} / \mathrm{N}(z 40)$ to be detected. This explains most likely the non-detection in previous observations.

From the observed WR emission lines we concluded that all WR regions (except NGC 3049) contain a mixed population of WNL, and early WC and/or WO3-4 stars. Weak CIII $\lambda 5696$ indicative of late WC stars is found in the high-metallicity WR region of NGC 3049. The estimated WC/WN number ratios (typically between $0.2-0.4$ ) show no clear trend with metallicity. For low-metallicity objects $\left(Z \simeq 0.20 \mathrm{Z}_{\odot}\right)$, these values are larger than the observed 
WC/WN ratios in Local Group objects with similar $Z$ (MJ98). We argue that our WC/WN values are compatible with expectations for regions of short star formation. For He2-10 and NGC 3049 the derived WC/WN ratio is below the trend given by MJ98. This can be due to the fact that we observe a short burst at a particular time (quite low probability), or that the number of WC stars is systematically underestimated. The solution awaits new observations and quantitative analysis of 'WC+WN galaxies' of different metallicities.

Detecting both WN and WC features provides a considerable improvement to derive burst parameters. Given the $\mathrm{WN} \rightarrow \mathrm{WC}$ sequence, which is expected to be followed only by the most massive WN stars, it is clear that the predictions of WC/WN populations are particularly sensitive to the evolutionary scenario and burst parameters (e.g., IMF, burst duration).

The comparisons of the observed EWs and line intensities relative to $\mathrm{H} \beta$ with models do not all show a simple picture. The most important effects and uncertainties which may affect such a comparison have been amply discussed in SCK99. In order to reproduce the WR lines, young populations with short durations of star formation are required. From our quantitative modeling, we find a conservative limit for burst durations of typically $\Delta t \lesssim 2-4 \mathrm{Myr}$. The majority of the observed quantities can be reproduced reasonably well by the SV98 models with a Salpeter IMF. Although some WR lines may indicate a flatter IMF in some regions, no clear case requiring a significantly flatter IMF is found. Much steeper IMF slopes may, however, not be compatible with our data. These results are in agreement with other studies of similar objects (see SCK99 and references therein).

\section{References}

Allen S.W. 1995, MNRAS 276, 947

Calzetti D. 1997, AJ 113, 162

Conti P.S. 1991, ApJ 377, 115

Heckman T.M., González-Delgado, R., Leitherer, C. et al. 1997, ApJ 482, 114

Maeder A., Meynet G. 1994, A\&A 287, 803

Mas-Hesse J.M., Kunth D. 1999, A\&A in press

Massey P., Johnson O. 1998, ApJ 505, 793 (MJ98)

Meynet G. 1995, A\&A, 298, 767

Schaerer D., Contini T., Kunth D. Meynet G., 1997, ApJ 481, L75

Schaerer D., Vacca W.D. 1998, ApJ 497, 618 (SV98)

Schaerer D., Contini T., Kunth D. 1999, A\&A 341, 399

Schaerer D., Contini T., Pindao, M. 1999, A\&AS 136, 35 (SCP99)

Steidel C.C., Adelberger, L.L., Giavalisco, M. et al. 1999, ApJ in press

Vacca W.D., Conti P.S. 1992, ApJ 401, 543 (VC92)

\section{Discussion}

Schmutz: Since I have seen the first spectra of WR galaxies back in 1989 I have always wondered that there are galaxies that have a stronger emission at N III 4650 than the 4680-blend. There is no WR star that has this characteristics and therefore, it is 
impossible that a mixture of WR stars could reproduce this feature. Do you have an explanation for this?

Contini: (1) In WR galaxies, the bump around $4640-4650 \AA$ is not stronger than He II 4686 but of the same intensity or even lower. (2) We do not detect the N III 4640 line but a blend of several broad emission lines from WR stars (e.g., N III 4640, C III 46404650). (3) WR-galaxies contain thousands of WR stars of different subtypes (WN, WC) which produce a variety of lines around $4640-4650 \AA$. So from my point of view, it is not surprising to observe blended lines around $4640-4650 \AA$ which have nearly the same intensity as the He II 4685 line.

Leitherer: In a few years we will hopefully have a large number of spectra of starforming galaxies at high redshift. What are the prospects of detecting spectral lines in the restframe UV lines which are unique signatures of WC stars?

Contini: I'm not an expert in UV spectroscopy but I guess that it should be possible in the future to detect clear signatures of WR stars in high-S/N rest-frame UV spectra of high-redshift starburst galaxies. Most common lines are those of C IV 1550, He II 1640 and, especially, C III 2293 which is prominent in WC stars but absent in WN stars.

Massey: One comment and a question. First, the correlation of WC/WN ratio with metallicity seen by Massey \& Johnson (1998) in the Local Group, as discussed yesterday, is presumably true only for populations that are well-mixed in age (i.e., number of stellar births equal to number of stellar deaths). But your models predict these things are very short lived births. So, the fact that your WC/WN ratio doesn't correlate with metallicity in the way the Local Group sample does isn't really a conflict. My question is now, do you allow for the C IV 5808 that comes from the WN stars themselves? As Walborn emphasized this morning, C IV 5808 is seen in the spectra of many WN stars. Do you allow for this, or what?

Contini: I agree that CIV 5808 is also seen in the spectra of WN stars, but their contribution is rather low compared to that in WC stars. Note that on average the C IV $5808 / \mathrm{He}$ II 4686 flux-ratio is only $\sim 0.05$ in WN stars.

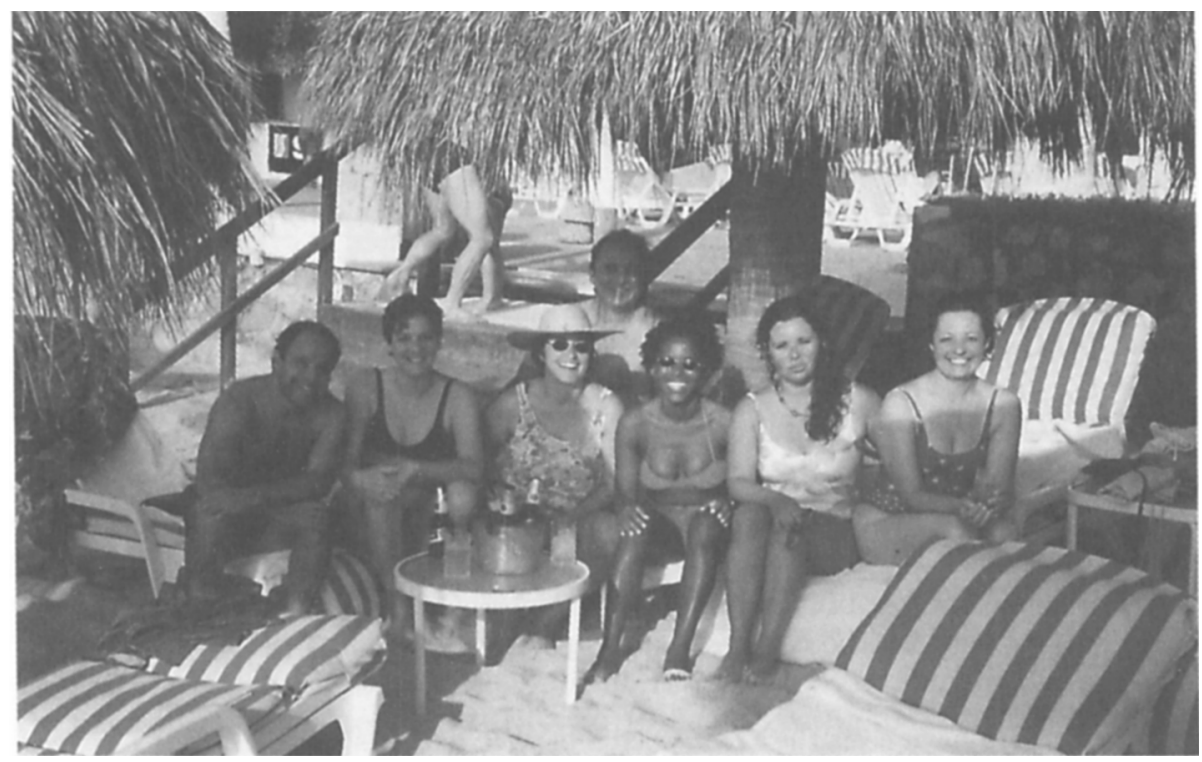

